EPJ Web of Conferences 113,01002 (2016)

DOI: $10.1051 /$ epjconf/201611301002

(C) Owned by the authors, published by EDP Sciences, 2016

\title{
Exploring the few- to many-body crossover using cold atoms in one dimension
}

\author{
Nikolaj Thomas Zinner ${ }^{1, a}$ \\ ${ }^{1}$ Department of Physics and Astronomy, Aarhus University, Ny Munkegade 120, DK-8000 Aarhus C, Den- \\ mark
}

\begin{abstract}
Cold atomic gases have provided us with a great number of opportunities for studying various physical systems under controlled conditions that are seldom offered in other fields. We are thus at the point where one can truly do quantum simulation of models that are relevant for instance in condensed-matter or high-energy physics, i.e. we are on the verge of a 'cool' quantum simulator as envisioned by Feynman. One of the avenues under exploration is the physics of one-dimensional systems. Until recently this was mostly in the many-body limit but now experiments can be performed with controllable particle numbers all the way down to the few-body regime. After a brief introduction to some of the relevant experiments, I will review recent theoretical work on one-dimensional quantum systems containing bosons, fermions, or mixtures of the two, with a particular emphasis on the case where the particles are held by an external trap.
\end{abstract}

\section{Introduction}

Atomic gases cooled to extremely low temperatures are a very rich and expanding field of physics where one may explore many different features of quantum few- and many-body systems in a highly controlled manner [1-4]. In these systems one has accurate control over both geometry and interactions, and a hotly pursued feature has been the realization of systems in one dimension (1D) [5-12]. The interactions are also controllable in 1D [13] and one may even get to the hard-core regime and study the Tonks-Girardeau limit $[14,15]$ in experiments $[7,8]$. In the latest developments it has now become possible to use $1 \mathrm{D}$ setups to reach the limit of small particle number [16] and few-body fermion systems have been realized in several experiments in the Jochim group in Heidelberg [1721]. A lot of theoretical research has been devoted to explore few-body physics in $1 \mathrm{D}$ and also to try to extrapolate into the many-body regime. Here I will review some of these developments with an excusable bias toward some of the work performed in our collaboration. Unless otherwise stated I will focus exclusively on 1D systems with short-range interactions that are implemented by a Dirac delta function. The basic Hamiltonian is

$$
H=\sum_{i}\left[\frac{p_{i}^{2}}{2 m}+V_{e}\left(x_{i}\right)\right]+\sum_{i<j} V\left(x_{i}-x_{j}\right),
$$

ae-mail: zinner@phys.au.dk 
where the sums run over the number of particles, $i=1, \ldots, N, x_{i}$ is the coordinate and $p_{i}$ the momentum of the $i$ th particle. The external trapping potential $V_{e}(x)$ is assumed to be the same for all particles. The mass $m$ is assumed to be the same for all particles in most of the discussion below, except for the section on mixtures where I will explicitly state when different masses are considered. The two-body interactions are given by terms of the form $V\left(x_{i}-x_{j}\right)=g \delta\left(x_{i}-x_{j}\right)$, where $g$ is the coupling strength. When I talk about strong interactions it is the limit where $1 / g \rightarrow 0$. For fermions the interactions are typically only between pairs of opposite spin projection. However, due to the Pauli principle enforcing the antisymmetry of pairs with identical spin projection, the interaction terms in the Hamiltonian above will have no consequence for identical fermion pairs. For bosons with more than one internal component, one has to consider the possibility of interactions between the pairs with the same internal components (intraspecies) and pairs with different internal components (interspecies). Intra- and interspecies interactions do not have to be the same in general. They will all be assumed to be given by Dirac delta functions. In the discussions below I will make it clear whenever this is important.

\section{Bosonic systems}

A particular focal point of attention in the work on few-boson systems has been the transition from weak to strong interactions. In the presence of strong short-range interactions, a pair of bosons will have a wave function that has to vanish when the two particles overlap. As noticed in the famous paper of Girardeau [15], this means that the ground state may now be described by a totally antisymmetric fermionic wave function if one makes appropriate changes of signs to ensure bosonic symmetry. For instance, if you take $N$ identical bosons with no internal degrees of freedom and put them into some trapping potential, then the ground state for strong interactions is obtained by taking the $N$ lowest single-particle eigenfunctions of the single-particle Schrödinger equation and construction their totally antisymmetry (Slater determinant) $N$-body wave function, and finally taking the norm of that to make sure that the wave function does not change sign under exchange of any two particles.

A number of studies have explored the physics of few-boson systems as the interactions are changed from weak to strong using the multi-configurational method [22-24] for double-wells and exact diagonalization [25-28] or diffusion Monte Carlo [29, 30] in a harmonic trap. In the latter case of a harmonic trap, Girardeau has given a number of exact solutions for strong interactions [31] that also apply for bosons with multiple internal states (spinor bosons). The fact that the exact solution of the two-body problem in a harmonic trap is available [32] has been used to propose analytical $N$-body states [33]. This is very similar to using Jastrow factors to incorporate correlations [34, 35].

In recent years, we have explored the physics of two-component Bose systems in harmonic traps, i.e. similar mass bosons with two internal degree of freedom that we denote $A$ and $B$. In the limit where the $A A$ and $B B$ interactions are negligible compared to $A B$ interactions, we found that one may solve the three-body problem exactly for strong interactions [36]. The ground state turns out to have an energy that is half an odd integer times the harmonic trap quantum, and its wave function (which is doubly degenerate) displays the precursor of ferromagnetism, i.e. the spatial ordering of the particles is either $A A B$ or $B A A$, but never $A B A$. On the other hand, one can find excited state in the system that only display the $A B A$ spatial ordering. In our latest work [37], we confirm that this kind of fractional energy behavior persists for larger systems and we present analytical results for the four-body state (two $A$ and two $B$ atoms) for strong interactions. Indeed, already for balanced ten particle systems with strong $A B$ interactions one can see the perfect ferromagnetic ordering emerge in the harmonic trap with a profile that approximates that expected in the many-body limit [37]. Another very recent study focusing on the strongly interacting limit also finds ferromagnetic behavior in this parameter regime [38]. 


\section{$21^{\text {st }}$ International Conference on Few-Body Problems in Physics}

\section{Fermionic systems}

The solution of the two-component Fermi system in 1D in known in the homogeneuos case from the nested Bethe ansatz of Gaudin and Yang [39]. However, in the case of a non-trivial confining potential no exact solution to the $\mathrm{N}$-body problem is known for general interaction strength. Here I will focus on harmonically trapped fermions but stress that many of the results and methods can be applied to other external confining geometries as well. An approach under exploration is to use Bethe ansatz in conjunction with a variational principle [40] while also the multi-configurational Hartree method has been applied [41] However, at this point it is safe to say that many studies rely in one way or another on exact diagonalization [42-49]. In the strongly interacting limit, some exact solutions are in fact known [50-52], although we caution that using the spin formalism of for instance Ref. [50] can lead to errors already at the level of four particles [43]. In our recent work on few-fermion physics, we have considered three-body problems both for two- [53] three-component systems [54]. A new variational approach was introduced in Ref. [53] which aims to find an accurate energy and wave function for the three-body problem using knowledge of the extreme limits of vanishing and infinite interaction strength. This provides a very accurate approximation that even extends to mass-imbalanced systems. Most recently, we have have considered dynamics of strongly interacting few-body systems in the case where one has time-dependent external traps. This may lead to dynamical engineering of magnetic quantum states [55].

The interesting question of how to get from the few- to the many-body limit has received much attention lately, in particular for strongly interacting particles. Again, Girardeau has written down some exact solutions to the two-component Fermi system in a trap with strong interactions [56] that are simple and beautiful, and have captured much attention. While they are of great theoretical use, they have little experimental relevance as they are superpositions of states that are adiabatically connected to experimentally relevant states for large but finite interaction strength. This was predicted in Ref. [57] and has recently been confirmed experimentally in Ref. [21]. Studies of the few- to many-body limit have been done using diffusion Monte Carlo [58], exact diagonalization [59, 60, 62], and most recently also coupled-cluster methods from quantum chemistry [63]. A particularly interesting feature is the fact that one may map the strongly interacting problem onto a spin model of the Heisenberg type [60-62], but with nearest-neighbor interactions that now depend on the external confinement and are not constant in general. It is interesting to note that the ansatz solution of the strongly interacting problem presented in Ref. [62] allows one to obtain a very accurate expression for the Heisenberg Hamiltonian in the case of harmonic confinement.

In our group we have pioneered the use of the Lee-Suzuki effective interaction method combined with exact diagonalization for $1 \mathrm{D}$ systems with short-range interactions [37, 59]. It provides a large reduction in computational time over traditional exact diagonalization with the bare Dirac delta function interaction. This has allowed us to consider for instance the polaron problem, i.e. a Fermi gas of one component (spin up) and a single (impurity) particle of the other component (spin down), for up to ten particles. Here one see a very characteristic phase separation with the impurity going to the middle of the trap causing a deep indentation in the majority particle density. This is also seen in the recent study of Ref. [62] where the many-body limit could be explored analytically using a very accurate ansatz wave function.

\section{Mixed systems}

Last but certainly not the least interesting are the mixed systems. This should be understood as those systems which are mixtures of fermions and bosons that may be either of equal or unequal mass. As in all the previous examples, Girardeau has once again been one of the first to consider such 
systems [64, 65]. In Ref. [64] a very interesting Bose-Fermi mapping was introduced. However, as we have pointed out in Ref. [57], these mappings are not useful for systems under non-trivial external confinement, and have to be applied with great care. While for three-body problems with equal mass Bose-Bose (spinor) mixtures one can indeed obtain the correct results for strong interactions [66], this does not extend to larger systems using Bose-Fermi mappings [57]. In Ref. [67] a four-body Bose-Fermi mixture with equal mass is considered in the strongly interacting regime, and the results are compared to a numerical study of the trapped system using the density-matrix renormalization group (DMRG) method. The Bose-Fermi mapping predicts a totally fermionized state in the strongly interacting limit that is identical to that obtained in a four-body system of identical (spinless or spinpolarized) fermions. This latter state has a characteristic four-hump structure in the harmonic trap. The DMRG calculations in Ref. [67] seem to confirm that this is indeed the strongly interacting ground state. However, applying the formalism of Ref. [57] one may confirm that this is not the case, and that the density akin to four spinless fermions is only found in an excited state. This leads one to suspect that the DMRG (built on a variational principle) may have gotten stuck in an excited state. This is plausible due to the (quasi)-degenerate manifold of state found in the strongly interacting limit [57]. This highlights the need for (semi)-analytical insight into the strongly interacting regime in spite of the success of the DMRG method over the past decades.

The problem of mixtures of Bose and Fermi atoms has already an intricate structure at the level of three atoms [54, 68, 69]. In particular, the strongly interacting limit and the (quasi)-degenerate manifold of state for large but finite interaction depend delicately on the composition, i.e. whether one considers two bosons and a fermion (all of equal mass) or vice versa $[68,69]$, or one can consider taking three altogether different components [54]. In light of this it is a very interesting question how can we determine which final state for strong interaction will be reach given an initial state for vanishing interaction strength? More precisely, if we adiabatically increase the interacting strength from zero to a very large value (effectively infinite), which state is reached? This question was explored in great detail in Ref. [70], and further elaborated by the same author in Ref. [71]. Using the symmetries of the problem and general mathematical arguments, Ref. [71] argues that for the case of four particles or less one may solve this issue by using the symmetries of the system. On the other hand, when the particle number is larger than four, one can no longer expect this and must resort to other means. This is an interesting question that certainly deserves further exploration.

Another important mixed system is the unequal mass case [72-76]. Some recent results have demonstrated a phase separation in the light component for Fermi-Fermi mixtures [75], which can also be seen for a single heavy impurity in a light Bose system [74]. In fact, the case of a single impurity in a Bose gas is often called the 'Bose polaron' problem and is much studied at present in the few-body limit $[73,74,76]$. In our recent work we have explored the dynamics when the bosonimpurity interaction is suddenly switched on. While the formalism used there works for general dimensions, we focused on the 1D case in Ref. [73] where this kind of quench protocol leads to oscillatory behavior in the weakly interacting regime. In Ref. [74] we present our results on the static configuration of the Bose polaron using a new formalism that is a hybrid of the hyperspherical adiabatic approach. What is particularly interesting about this latter work is the fact that one can extrapolate to large particle numbers with miniscule computational effort. In fact, as we argue in Ref. [74] we expect the approximations we make to work better for larger systems. By comparison to numerically exact results [37] for systems with ten particles or less we confirm that the (semi)analytical method put forth in Ref. [74] is accurate within a few percent already for eight bosons and an impurity. In addition, this new formalism can handle any trapping potential that can be different for the bosons and the impurity, it can tackle any mass ratios, and the traps may even be displaced from one another. 


\section{Outlook}

As I have argued above, the experimental situation is very promising for studying few-body physics in $1 \mathrm{D}$, as well as for gaining knowledge about how one extrapolates to the many-body regime. I like to call this few-body physics in a many-body world. A considerable body of theoretical work has been put forth in this area and more is certainly to come. Many new avenues are being explored and I believe that new aspects of $1 \mathrm{D}$ physics will be found. I think that an important outstanding question concerns the notion of strong in real life. What does 'strong interaction' really mean? Above I have used the mathematical definition which means that you take a coupling constant to infinity. Some recent experimental results [21] strongly suggest that we understand that limit [57]. This was done with relatively few particles and I would really like to see how things change as we increase the number of particles, preferably one at a time. I am also very interested in exploring dynamics in 1D systems with strong interactions such as quantum state transfer [61] and dynamical quantum magnetic effects [55]. An intriguing question that comes to mind in this respect is that of spin-charge separation. It is know from 1D Luttinger liquid formalism that the low-energy excitations of a two-component Fermi gas in 1D split into charge density and spin density waves, and furthermore that these waves have different velocities, hence we get spin-charge separation. As far as I know, at this point it has not been unambiguously observed in electronic systems such as nanowires. What I would really like to see is how this effect emerges from a few-body perspective.

\section{Acknowledgements}

I thank my collaborators Artem Volosniev, Amin Dehkharghani, Oleksandr Marchukov, Dmitri Fedorov, Aksel Jensen, Manuel Valiente, David Petrosyan, Jonathan Lindgren, Christian Forssén, Jimmy Rotureau, and HansWerner Hammer. The experimental group of Selim Jochim in Heidelberg is gratefully acknowledged for discussions and for making their data available to us. This work is supported in part by the Danish Council for Independent Research DFF and the DFF Sapere Aude program.

\section{References}

[1] I. Bloch, J. Dalibard, and W. Zwerger, Rev. Mod. Phys. 80, 885 (2008).

[2] M. Lewenstein et al., Adv. Phys. 56, 243 (2007).

[3] T. Esslinger, Ann. Rev. Cond. Mat. Phys. 1, 129 (2010).

[4] N. T. Zinner and A. S. Jensen, J. Phys. G:Nucl. Part. Phys. 40, 053101 (2013).

[5] H. Moritz, T. Stöferle, M. Köhl, and T. Esslinger, Phys. Rev. Lett. 91, 250402 (2003).

[6] T. Stöferle, H. Moritz, C. Schori, M. Köhl, and T. Esslinger, Phys. Rev. Lett. 92, 130403 (2004).

[7] T. Kinoshita, T. Wenger, and D. S. Weiss, Science 305, 1125 (2004).

[8] B. Paredes et al., Nature 429, 277 (2004).

[9] T. Kinoshita, T. Wenger, and D. S. Weiss, Nature 440, 900 (2005).

[10] E. Haller et al., Science 325, 1224 (2009).

[11] E. Haller et al., Nature 466, 597 (2010).

[12] G. Pagano et al., Nature Phys. 10, 198 (2014).

[13] M. Olshanii, Phys. Rev. Lett. 81, 938 (1998).

[14] L. W. Tonks, Phys. Rev. 50, 955 (1936).

[15] M. D. Girardeau, J. Math. Phys. 1, 516 (1960).

[16] F. Serwane et al., Science 332, 336 (2011). 
[17] G. Zürn et al., Phys. Rev. Lett. 108, 075303 (2012).

[18] G. Zürn et al., Phys. Rev. Lett. 111, 175302 (2013).

[19] A. Wenz et al., Science 342, 457 (2013).

[20] S. Murmann et al., Phys. Rev. Lett. 114, 080402 (2015).

[21] S. Murmann et al., arXiv:1507.01117 (2015).

[22] S. Zöllner, H.-D. Meyer, and P Schmelcher, Phys. Rev. A 74, 063611 (2006); Phys. Rev. A 75, 043608 (2007); Phys. Rev. Lett. 100, 040401 (2008).

[23] E. Tempfli, S. Zöllner, and P. Schmelcher, New J. Phys. 11, 073015 (2009).

[24] S. Zöllner, H.-D. Meyer, and P Schmelcher, Phys. Rev. A 78, 013629 (2008);

[25] F. Deuretzbacher, K. Bongs, K. Sengstock, and D. Pfannkuche, Phys. Rev. A 75, 013614 (2007).

[26] M. A. Garcia-March and Th. Busch, Phys. Rev. A 87, 063633 (2013).

[27] S. Campbell, M. A. Garcia-March, T. Fogarty, and Th. Busch, Phys. Rev. A 90, 013617 (2014).

[28] M. A. Garcia-March et al., Phys. Rev. A 90, 063605 (2014).

[29] M. A. Garcia-March et al., Phys. Rev. A 88, 063604 (2013).

[30] M. A. Garcia-March et al., New J. Phys. 16, 103004 (2014).

[31] M. D. Girardeau, Phys. Rev. A 83, 011601(R) (2011).

[32] T. Busch, B.-G. Englert, K. Rzążewski, and M. Wilkens, Found. Phys. 28, 549 (1998).

[33] I. Brouzos and P. Schmelcher, Phys. Rev. Lett. 108, 045301 (2012).

[34] I. Brouzos and A. Förster, Phys. Rev. A 89, 053632 (2014).

[35] B. Wilson, A. Förster, C. C. N. Kuhn, I. Roditi, and D. Rubeni, Phys. Lett. A 378, 1065 (2014).

[36] N. T. Zinner et al., Europhys. Lett. 107, 60003 (2014).

[37] A. S. Dehkharghani et al., Scientific Reports 5, 10675 (2015).

[38] P. Massignan, J. Levinsen, and M. M. Parish, arXiv:1507.02814 (2015).

[39] M. Gaudin, Phys. Lett., Sect. A 24, 55 (1967); C. N. Yang, Phys. Rev. Lett. 19, 1312 (1967).

[40] D. Rubeni, A. Förster, and I. Roditi, Phys. Rev. A 86, 043619 (2012).

[41] I. Brouzos and P. Schmelcher, Phys. Rev. A 87, 023605 (2013).

[42] P. O. Bugnion and G. J. Conduit, Phys. Rev. A 87, 060502(R) (2013).

[43] S. E. Gharashi and D. Blume, Phys. Rev. Lett. 111, 045302 (2013).

[44] T. Sowiński, T. Graß, O. Dutta, and M. Lewenstein, Phys. Rev. A 88, 033607 (2013).

[45] P. D’Amico and M. Rontani, J. Phys. B 47, 065303 (2014); Phys. Rev. A 91, 043610 (2015).

[46] S. E. Gharashi, X. Y. Yin, and D. Blume, Phys. Rev. A 89, 023603 (2014).

[47] R. Lundmark, C. Forssén, and J. Rotureau, Phys. Rev. A 91, 041601(R) (2015).

[48] S. E. Gharashi, X. Y. Yin, and D. Blume, Phys. Rev. A 91, 013620 (2015).

[49] T. Sowiński, M. Gajda, and K. Rza̧żewski, Europhys. Lett. 109, 26005 (2015).

[50] L. Guan, S. Chen, Y. Wang, and Z.-Q. Ma, Phys. Rev. Lett. 102, 160402 (2009).

[51] C. N. Yang, Chin. Phys. Lett. 26, 120504 (2009).

[52] X. Cui and T.-L. Ho, Phys. Rev. A 89, 023611 (2014).

[53] N. J. S. Loft et al., Eur. Phys. J. D 69, 65 (2015).

[54] A. G. Volosniev et al., Few-Body Syst. 55, 839 (2014).

[55] A. G. Volosniev, H.-W. Hammer, and N. T. Zinner, arXiv:1507.00186 (2015).

[56] M. D. Girardeau, Phys. Rev. A 82, 011607(R) (2010).

[57] A. G. Volosniev et al., Nature Commun. 5, 5300 (2014).

[58] G. E. Astrakharchik and I. Brouzos, Phys. Rev. A 88, 021602(R) (2013).

[59] E. J. Lindgren et al., New J. Phys. 16, 063003 (2014). 
[60] F. Deuretzbacher et al., Phys. Rev. A 90, 013611 (2014).

[61] A. G. Volosniev et al., Phys. Rev. A 91, 023620 (2015).

[62] J. Levinsen, P. Massignan, G. M. Bruun, and M. M. Parish, Science Adv. 1, e1500197 (2015).

[63] T. Grining et al., arXiv:1507.03174 (2015); arXiv:1508.02378 (2015).

[64] M. D. Girardeau and M. Olshanii, Phys. Rev. A 70, 023608 (2004).

[65] M. D. Girardeau and A. Minguzzi, Phys. Rev. Lett. 99, 230402 (2007).

[66] F. Deuretzbacher et al., Phys. Rev. Lett. 100, 160405 (2008).

[67] B. Fang, P. Vignolo, M. Gattobigio, C. Miniatura, and A. Minguzzi, Phys. Rev. A 84, 023626 (2011).

[68] N. L. Harshman, Phys. Rev. A 86, 052122 (2012).

[69] R. E. Barfknecht, I. Brouzos, A. Förster, Phys. Rev. A 91, 043640 (2015).

[70] N. L. Harshman, Phys. Rev. A 89, 033633 (2014).

[71] N. L. Harshman, arXiv:1501.00215 (2015); arXiv:1505.00659 (2015).

[72] N. P. Mehta, Phys. Rev. A 89, 052706 (2014).

[73] A. G. Volosniev, H.-W. Hammer, and N. T. Zinner, Phys. Rev. A 92, 023623 (2015).

[74] A. S. Dehkharghani, A. G. Volosniev, and N. T. Zinner, arXiv:1503.03725 (2015).

[75] D. Pęcak, M. Gajda, and T. Sowiński, arXiv:1506.03592 (2015).

[76] N. P. Mehta, arXiv:1508.00156 (2015). 